\title{
The relationship between plasma corticosterone levels and leverpress avoidance vs. escape behaviors in rats
}

\author{
DAVID F. BERGER, JAMES J. STARZEC, and ELLIOTT B. MASON \\ State University of New York College at Cortland, Cortland, New York 13045
}

\begin{abstract}
Groups of rats were exposed to training procedures that produce leverpress avoidance (Experiment 1) or escape (Experiment 2) behaviors. Plasma samples were obtained by decapitation after 30 sessions, and corticosterone concentrations were determined and compared with those of corresponding yoked and nonshocked (time-in-box) groups. The mean corticosterone level of the "avoiders" was lower than that of their yoked mates and not different from that of their nonshocked mates. This indicated a reduction in their arousal level with mastery of the avoidance response, which could not be accounted for by the reduction in numbers of shocks received. The mean corticosterone level of the "escapers" was as high as that of their yoked mates, and both levels were higher than that of the nonshocked group.
\end{abstract}

The activation of the pituitary-adrenal system in response to stress in general (Selye, 1956) and to the avoidance situation in particular has been established and discussed by several authors (see Brush \& Froelich, 1975; Di Giusto, Cairncross, \& King, 1971; Gray, 1971; Leshner, 1978; Levine, 1971). Coover, Ursin, and Levine (1973) proposed that graded elevations in plasma corticosterone follow aversive stimulation and can serve as an appropriate indicator of the associated arousal levels of the animal. They took three postsession plasma samples from their rats during the course of shuttle-box avoidance training and found elevations of plasma corticosterone levels at the beginning of training that decreased later when their animals' avoidance behavior had stabilized. Coover et al. (1973) argued that the indicated decrease in the arousal level of their rats was not necessarily due to a reduction in the frequency of electric shocks associated with good avoidance performance. In support of their position, they found no significant correlation between the number of shocks received during the sampled sessions and the corresponding plasma corticoid levels of the animals. They also found that increases in the level of the hormone could be obtained following periods of no shocks (i.e., forced extinction). Coover et al. (1973)

This research was supported by Grants 76883 from the American Heart Association and 76-UP16 from the Heart Association of Upstate New York, Inc., and the Finger Lakes Chapter. Sincere appreciation must be extended to: F. R. Brush and W. DeVito, for their constructive suggestions; W. DeVito, R. Hesse, and D. Pytko, for running the experiments; and L. Dewey and C. Forte for analyzing the plasma samples. Requests for reprints should be sent to David F. Berger, Psychology Department, State University of New York, Cortland, New York 13045. therefore suggested that the avoidance situation becomes less stressful as the rats achieve predictability and control of it.

However, several issues remain unresolved. For example, the absence of a correlation between the number of shocks and corticosterone levels could be due to the reported high variability of the latter (Davis, Porter, Livingstone, Herrmann, MacFadden, \& Levine, 1977; Weiss, 1971). Also, Coover et al. (1973) did not separate such factors as habituation, adaptation, or sensitization to the shocks from those associated with mastery of the avoidance response.

A clarification of the effects of the reduction in the number of shocks received as a consequence of avoidance responding, as well as the possible effects of habituation, adaptation, or sensitization are not available from the correlational approach. The use of a yoked-control procedure, in which another rat receives the same number and duration of shocks as its counterpart in the escape/avoidance situation, is required. The need for data from the use of the yokedcontrol procedure was also noted by Natelson, Krasnegor, and Holaday (1976).

In addition, Coover et al. (1973) did not report on the corticosterone levels of those rats that did not learn to avoid (presumably, about 28\%; see Brush, 1966). Fjermestad (1976) conducted an experiment similar to that of Coover et al. (1973) but used the leverpress avoidance situation. Following the initial elevations, he found similar decreases in plasma corticosterone levels to be associated with successful avoidance acquisition. However, no such significant decreases in stress hormone levels occurred in rats failing to acquire the avoidance response, whether by self-selection or in a group trained with conditions 
that generally produce poor avoidance performance (see Berger \& Brush, 1975). Only those animals that learned to avoid $(60 \%$ or more avoidances), regardless of the training conditions, showed reduced stress hormone levels at the end of the training when the behaviors had stabilized. Fjermestad also found no significant correlations between corticosterone levels and numbers of shocks received.

Finally, Coover et al. (1973) and Fjermestad (1976) obtained repeated samples of plasma from their rats following several selected sessions during the course of their escape/avoidance training. Under such conditions, it is conceivable that the extra time required for anesthesia with highly aroused animals may have allowed the stress of the sampling procedure to alter the plasma corticosterone levels. Leshner (1978, p. 255) and others have reported that a rise in glucocorticoid secretion is detectable within 3-5 min after the onset of a stressor. Therefore, a single sampling by decapitation is preferable because it minimizes any possible reaction by the rapidly responding pituitary-adrenal system from the sampling per se.

\section{EXPERIMENT 1}

With this experiment, we attempted to replicate the reduction in plasma corticosterone levels that accompanies mastery of an avoidance response (Coover et al., 1973; Fjermestad, 1976), but with the addition of a yoked-control group to examine the effects of the reduced number of shocks received as a consequence of the avoidance behavior of the experimental animals. The possible effects of any type of alteration in the sensitivity of the rats that may result from the prolonged exposure to the shocks could also be detected. Escape/avoidance training using the successful 60-sec signal-shock interval (see Berger \& Brush, 1975, Experiment 2) was presented for 30 sessions to be certain that the animals' behavior had stabilized prior to the plasma sampling by decapitation.

\section{Method}

Subjects. Forty-eight adult male Sprague-Dawley albino rats were obtained from Taconic Farms, Germantown, New York. All were experimentally naive and weighed between 245 and $370 \mathrm{~g}$ upon arrival. The animals were maintained on a cholesterolsupplemented diet (Lasser, Roheim, Edelstein, \& Eder, 1973), because they were also part of our investigation for the American Heart Association of the effects of psychological stress on lipid metabolism. The diet consisted of $1 \%$ cholesterol, $10 \%$ olive oil, and $89 \%$ Purina Lab Chow. The colony room was continuously illuminated.

Apparatus. Four BRS-Foringer Series 900 and two BRS/LVE Model 143 operant conditioning chambers were used. Three of the six chambers (two BRS-Foringer and one BRS/LVE) were programmed for the escape/avoidance conditions. The other three were used for the yoked-control treatment and therefore had no levers or signals. The shock source for each of the yoked chambers was cued by the shock programmer for one of the adjacent escape/ avoidance chambers. Each chamber was enclosed in an outer box for attenuation of external sounds. The warning signal for all appropriate boxes was a $1,000-\mathrm{Hz}$ tone provided by a BRS-Foringer AU-902 audio generator. It raised the 72-dB noise level, from the ventilating fan in each box, to $79 \mathrm{~dB}$ (SPL).

In the BRS-Foringer boxes, the safety signal came from pulsing 28-V dc through two 14-V (GE 1893) bulbs wired in series at $1 \mathrm{~Hz}$. These bulbs were located over the center of the conditioning chambers on the ceiling of their outer boxes. Their grid shocks came from BRS-Foringer SC-901 shock scramblers and were generated by BRS-Foringer SG-901 shock sources. The safety signal for the BRS/LVE box was provided by pulsing a single 28-V (GE 1820) bulb, located at the top of the lever panel, with $28-\mathrm{V}$ dc at $1 \mathrm{~Hz}$. Their grid shocks came from BRS/LVE SG-903 shock generators through BRS/LVE SC-922 shock scramblers.

For all the boxes, a shock intensity of $2 \mathrm{~mA}$ was set by placing an ac milliammeter across adjacent grid bars. General illumination in each chamber was provided by a 28-V (GE 1820) cue light, $5 \mathrm{~cm}$ over the lever, which was operated at $20-\mathrm{V}$ ac from an isolated source. Each lever required an operating force of $12 \mathrm{~g}$.

Procedure. The animals were assigned randomly to one of the three groups. The experimental group (E) contained 16 rats. Each animal was assigned a yoked-control mate (Group Y) that was run at the same time as its experimental mate in an adjacent chamber. The rats in Group Y received unpredictable, uncontrollable grid shocks in the same sequence, order, and duration as did their escape/avoidance mate (when it failed to avoid), because their shock sources were cued together. Finally, a group of 16 animals served as a time-in-box control (Group TIB) for any effects due to daily handling and box exposure. These animals spent the same amount of time as all the others in the apparatus but without any signals or shocks. This triplet design is similar to that used by Weiss (1971).

Four days after arrival, the stressed animals (Groups E and Y) were placed on the cholesterol-supplemented diet, and they remained on it for 15 days prior to, as well as throughout, the escape/ avoidance training period. Our prior abservations had shown that the stressed groups ate significantly less of the diet than did the time-in-box control group. Therefore, Group TIB was started on the diet and run 1 day behind the others throughout the experiment. They were fed an amount equal to the mean intake of the other two animals in their triplet (which, as a group, ate essentially the same amounts) for the previous day. They also remained on the diet for 15 days prior to, as well as throughout, the training period. No food was available for any group during the daily training sessions.

On the day before the escape/avoidance training sessions began, the animals were allowed to explore their experimental chambers for $51 \mathrm{~min}$ with only the house lights and fans operating. The typical training trial began with the onset of the warning signal, which lasted for $60 \mathrm{sec}$. At the end of this interval, if no avoidance leverpress occurred, a .5-sec shock was delivered to the rat via the grid floor. Additional shock pulses were presented on a 1-min variable interval schedule, in the presence of the warning signal, until a leverpress did occur. A press during the warning signal, but before the first shock pulse, was recorded as an avoidance response, whereas a response following the occurrence of one or more shock pulses was classified as an escape response. The consequences of the press were the same in each case. A leverpress terminated the warning signal and the train of shock pulses if it had been initiated and started a 5-min safe period accompanied by the flashing-light safety signal. An escape response occurring during any .5-sec shock pulse could reduce its duration. The number of shock pulses received was recorded.

The 51-min daily training sessions held the duration of stress exposure constant and allowed for a maximum of 10 trials to occur, but the actual number in each session depended on the animal's response latencies. It was possible for an animal to spend the entire session in the presence of the initial warning signal, and, if the rat 
failed to respond, it would receive approximately 51 of the .5 -sec shock pulses. The last trial of the day could be terminated at any time by the end of the session, leaving the animals in the presence of the houselight and fan until they were removed from the chambers.

Two sets of triplets were run during each daily session, beginning at 8 a.m. and ending at approximately 4 p.m. This arrangement generally insured that a representative from each group was run during every session throughout each day, in an attempt to balance out differences in steroid levels associated with the time of the session. The animals in Group TIB were distributed among all the experimental chambers to control for any differences attributable to the apparatus alone, and they were exposed to only the houselight and ventilating fan during the training sessions.

Directly following their 30th session, the rats were removed from the operant chambers in random order. Upon removal, each was weighed, carried to an adjoining room, and decapitated immediately, and its trunk blood was collected in heparinized, soap-free tubes. The plasma was separated and frozen for later analysis (blind) of its corticosterone content by the fluorometric method of Glick, Von Redlich, and Levine (1964), as modified by Brush (Note 1).

\section{Results}

Group E's mean percentages of avoidance responses, based on the individual animal's five-session block medians, are presented in Figure 1 (solid lines) for comparison purposes. Overall, over $60 \%$ was obtained by the third block; mean performance climbed to over $70 \%$ during the last two blocks. A one-way within analysis of variance showed that the percentage of avoidances increased significantly over the training period $[\mathrm{F}(5,75)=17.07, \mathrm{p}<.05]$. A NewmanKeuls test showed that the percentage of avoidances in all subsequent blocks was greater than in Block 1, and that Blocks 5 and 6 were also higher than Block 2 (ps $<.05)$. The total percentage of avoidances for the 30 training sessions was obtained for each rat, based on its total number of trials. The group median of these total percentages was $69.9 \%$.

The mean corticosterone levels obtained from the plasma samples are illustrated in Figure 2. One sample from Group TIB was lost during centrifugation. The lighter bars in the left panel, labeled " 60 -sec condition," show the terminal plasma corticosterone levels ( \pm standard error) of Group E, Group Y, and Group TIB. A one-way analysis of variance revealed that there was a significant treatment effect $[F(2,44)$ $=9.57, \mathrm{p}<.05$ ]. Newman-Keuls comparisons showed that the mean corticosterone level of Group $E$ was lower than that of Group Y $(p<.05)$, and that both were higher than Group TIB (ps $<.05$ ).

Only 10 of the rats actually mastered the avoidance response (defined as $60 \%$ total avoidance and above). The mean plasma corticosterone level of this subgroup of "avoiders," together with those of their yoked and time-in-box control mates, are shown by the darker bars in the left panel of Figure 2. A oneway analysis of variance, recomputed with just these data, yielded a significant treatment effect $[F(2,27)=$ $5.31, \mathrm{p}<.05]$. The corticosterone levels of the

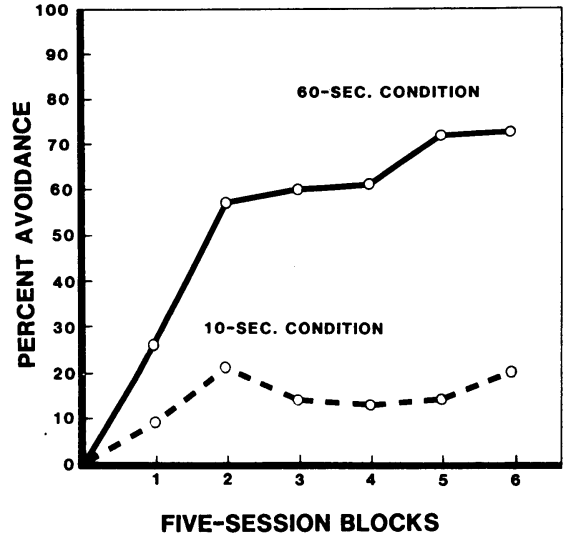

Figure 1. Percent avoidance responses as a function of the means of five-session block medians. The solid lines represent the performance of the group trained with a 60 -sec signal-shock interval (Experiment 1). The dashed lines represent the performance of the group trained with the 10-sec signal-shock interval (Experiment 2).

"avoiders" and their time-in-box control mates both differed from those of the yoked-control animals (ps $<.05$, Newman-Keuls), but not from each other. The mean hormone level of the six animals that attained totals of less than $60 \%$ avoidances (mean $=$ $28.2 \%$ ) was $29.48 \pm 5.44 \mathrm{mg} / 100 \mathrm{ml}$.

The total number of shocks received by each rat in Groups $\mathrm{E}$ and $\mathrm{Y}$ over the 30 sessions was obtained. The mean for the entire group was 218.8 .

\section{Discussion}

The 60 -sec signal-shock interval produced generally reliable leverpress avoidance behavior in Group E. The associated lower plasma corticosterone levels of Group E, compared with those of their yoked-control mates in Group Y, indicated a decrease in their arousal in relation to their mastery of the leverpress avoidance task. With the data from the poorer performers (below $60 \%$ total avoidances) excluded, the effect was even more pronounced. In fact, the mean plasma corticosterone levels of the "avoiders" were not significantly above those of their time-in-box control mates that received no shocks. Furthermore, the lower hormone levels cannot simply be the result of receiving fewer shocks or of some type of habituation, because such effects would have also reduced the corticosterone levels of Group Y.

These findings agree with those of Coover et al. (1973), who used the shuttle box. They are supported by those of Fjermestad (1976) with the leverpress procedure and, most recently, by those of DeVito (1979), who observed a gradual reduction in plasma corticosterone over training sessions using a procedure similar to the present one, but with a 30-sec signal-shock interval. Such results also reflect the apparent "nonchalance" of animals after they have 


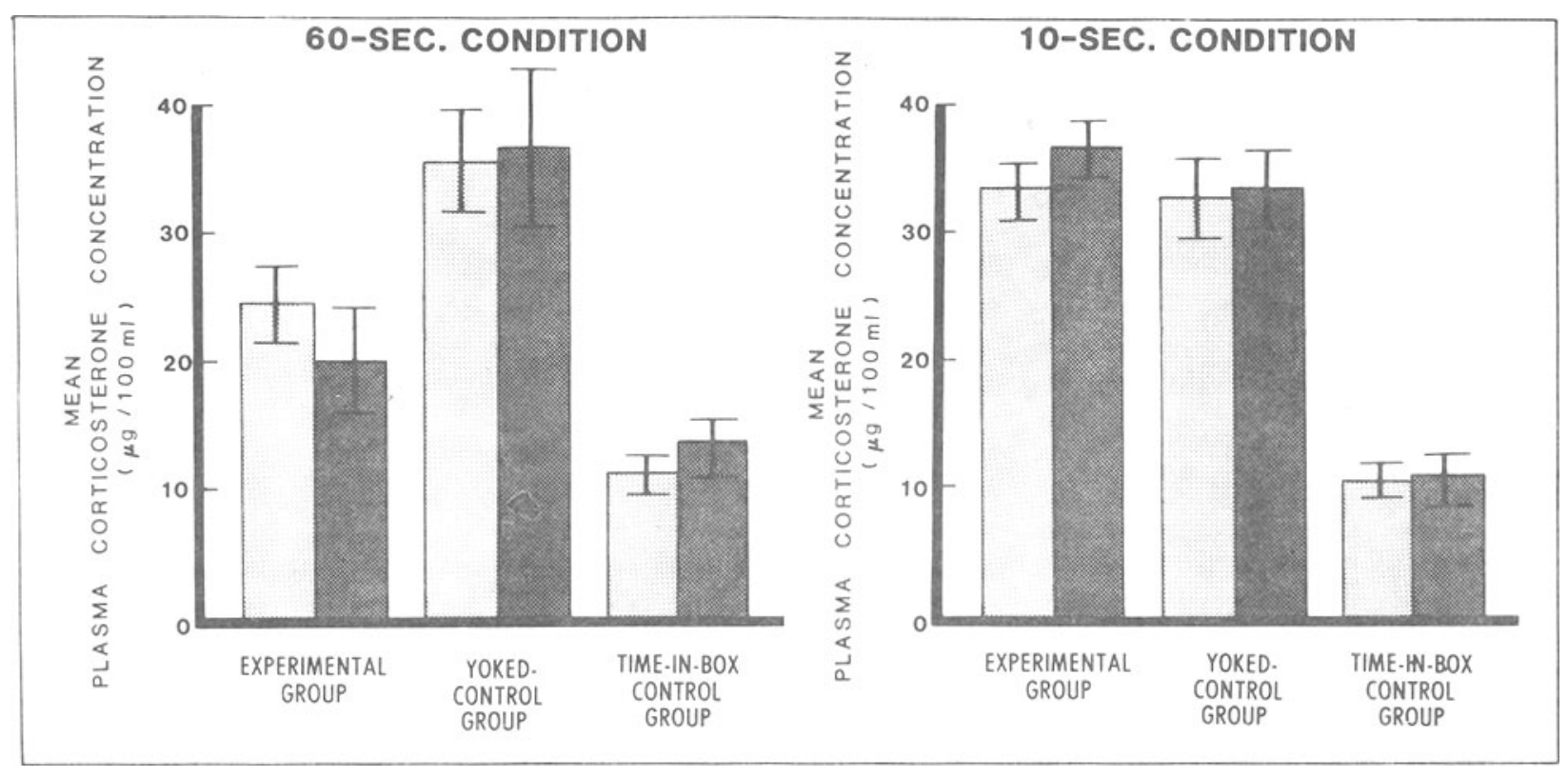

Figure 2. The concentrations of corticosterone obtained from plasma samples taken immediately following the 30th escape/avoidance training session. The three lighter bars in the left panel show the mean levels of the entire group trained with the 60-sec signal-shock interval condition (Experiment 1), the yoked-control group, and the time-in-box control group. The three darker bars in the left panel represent the mean corticosterone levels of the subgroup "avoiders" that obtained $60 \%$ or more avoidances and their yoked and timein-box mates, respectively. Similarly, the lighter bars in the right panel show the mean corticosterone levels of the entire group trained with the 10-sec signal-shock interval condition (Experiment 2) and those of the two corresponding control groups. The darker bars in the right panel show the mean corticosterone levels of the subgroup of "escapers" that obtained $30 \%$ avoidances or below and their yoked and time-in-box mates, respectively.

mastered an avoidance response, which has been commented on by Coover et al. (1973), Solomon and Wynne (1954), and others.

\section{EXPERIMENT 2}

The second experiment was aimed at measuring the terminal plasma corticosterone concentrations of rats that fail to acquire the leverpress avoidance response. The hormone levels of those self-selected six animals in Experiment 1 that avoided the initial brief shocks less than $60 \%$ of the time were relatively higher than those of the rats that mastered the avoidance task. However, by using the same escape/avoidance training procedure, but with a 10 -sec signal-shock interval, it was possible to insure that most of the rats would remain in the escape phase (Berger \& Brush, 1975). The same control conditions as in Experiment 1 were used again for the same reasons as before.

\section{Method}

Subjects. Forty-eight adult male rats of the same strain and weight range as those in Experiment 1 were obtained from the same source. They were also maintained on the same diet.

Apparatus. The apparatus was the same as that used in Experiment 1 above.

Procedure. The triplet design (Weiss, 1971) was used. Every detail of the procedure was as described for Experiment 1, except that the experimental group (E) was trained with a 10-sec signal-shock interval.

\section{Results}

The group mean percentages of avoidance responses of five-session medians for Group $\mathrm{E}$ are also presented in Figure 1 (broken lines). Their performance was relatively stable over the entire training period. They were generally unable to avoid most of the shocks, although they did efficiently escape from them. These data were analyzed as in Experiment 1. The results yielded a significant effect $[F(5,75)=12.23$, $\mathrm{p}<.05$ ]. However, the Newman-Keuls comparisons showed that the effect was due to the fact that the percentages of avoidances in Blocks 2 and 6 were significantly above those in Block 1 (ps $<.05$ ) and not to a general increase over trial blocks as in Experiment 1 . None of the other blocks differed from each other $(p>.05)$. The total percentage of avoidances for the 30 training sessions was again obtained for each rat, based on its total number of trials. The median of these total percentages was $\mathbf{1 5 . 2 \%}$.

The mean corticosterone levels obtained from the terminal plasma samples are illustrated by the lighter bars in the right panel of Figure 2, under the "10-sec condition" heading. A plasma sample from Group TIB was lost through a procedural error. A one-way analysis of variance indicated that there was a signifi- 
cant treatment effect $[F(2,44)=21.80, p<.05]$. Newman-Keuls comparisons showed that the corticosterone concentrations of Groups $E$ and $Y$ did not differ from each other $(p>.05)$, but were both higher than those of Group TIB (ps $<.05$ ).

Not all the rats failed to acquire the avoidance response. Thirteen were identified as having remained in the escape phase of training (defined as $30 \%$ total avoidances or below). The plasma corticosterone concentrations of these "escapers" were compared with those of their associated control mates by another one-way analysis of variance $[\mathrm{F}(2,35)=20.58, \mathrm{p}<.05]$. Once again, Newman-Keuls tests showed that the stress hormone levels of Groups $E$ and $Y$ were statistically equivalent $(p>.05)$ and higher than those of Group TIB (ps < .05). The levels of these subgroups are shown by the darker bars in the right panel of Figure 2. The remaining three animals in Group E attained above $30 \%$ total avoidances (mean $=42.8 \%$ ) and had a mean plasma corticosterone concentration of $19.10 \pm 3.62 \mathrm{mg} / 100 \mathrm{ml}$.

The total number of shocks received by each animal in the shocked groups during the entire 30 sessions was also tabulated. The overall mean was 385.9.

\section{Discussion}

As expected, the 10-sec signal-shock interval condition continued to disrupt the leverpress avoidance performance of most of the rats for the extended period of training, resulting in an average of less than $16 \%$ avoidances over the 30 sessions. In addition, as indexed by their corticosterone concentrations on the last day, the arousal levels of Group E were as high as those of their yoked-control counterparts in Group Y. Even though the rats in Group E had learned to escape from the train of shock pulses, the onset of the initial shock pulses at the end of the signal-shock interval was, for them, essentially uncontrollable, and their corticosterone concentrations reflect high arousal levels similar to those of their helpless (yoked) partners (see Weiss, 1971).

Interestingly, the mean level of Group $Y$ in the present experiment was actually slightly lower than that of the corresponding group (Y) in Experiment 1 that received considerably fewer shocks, thereby ruling out the numbers of shocks received as the determiner of the animals' corticosterone response. However, the corticosterone levels of the animals in Group E in both experiments were roughly inversely related to their levels of avoidance performance.

The above findings do not appear to agree with those of Davis et al. (1977), who found a decrease in the plasma corticosterone concentrations of rats after 15 days of unsignaled leverpress escape training, similar to that reported by Coover et al. (1973) with rats in the shuttle-box avoidance task. However, there are a number of important methodological differ- ences between the present and the Davis et al. (1977) studies that complicate attempts to compare them. The decrease in corticosterone level reported by the latter authors was based upon within-subjects comparisons with previous elevations earlier in the training, rather than in comparison with yoked-control animals, as in the present study. Davis et al. (1977, Experiment 2) did provide a group of rats with inescapable shocks, equated in number, distribution, and mean duration to those received by the rats in their first experiment. However, that procedure was not exactly comparable to the present yoked-control procedure because the .25 -sec shocks were presented on a fixed-interval 30-sec schedule and were therefore temporally predictable. Weiss (1970) reported lower plasma corticosterone level with predictable, as compared with unpredictable, shocks in yoked pairs of rats. The corticosterone data were reported by Davis et al. (1977) in terms of percentage change from the animals' mean basal levels, and those mean basal values were quite different from each other. Therefore, we cannot determine if the animals in Experiments 1 and 2 of Davis et al. (1977) differed significantly in corticosterone level on the 15th day of their training, and that information was not provided by the authors. Finally, the intensity and duration of the shocks differed between the present study and that of Davis et al. (1977). The latter used .4-mA shocks averaging $.25 \mathrm{sec}$ in duration, whereas $2-\mathrm{mA}, .5-\mathrm{sec}$ shocks were used in the present study.

It is not possible to determine from the present experiments whether the elevations in plasma corticosterone observed with Group E in Experiment 2 were the result of their failure to learn the avoidance response or the cause of that failure. Support for the latter position comes from several theorists who have proposed that the relationship between arousal and performance is nonmonotonic (e.g., see Ferguson, 1976, chap. 6). It is possible that the animals in that group were sufficiently aroused to be on the down side of an inverted- $U$ function. One possible interpretation would then be that the elevation in endogenous corticosterone observed may have actually been causally related to the poor avoidance performance. There is ample evidence that shows that exogenous corticosterone and related steroids reduce fear-motivated responding (e.g., Weiss, McEwen, Silva, \& Kalkut, 1969, 1970, with adrenalectomized rats), and they facilitate extinction of avoidance behavior (e.g., Leshner, 1978, p. 262; Wimersma Greidanus, 1970, with intact rats). Furthermore, Delft (1970) showed that pretraining and stress levels of endogenous corticosterone were inversely related to the rats' ability to learn the avoidance response in the pole-jump situation, and also that fewer intertrial responses were associated with the higher hormone levels. In their review, DiGiusto et al. (1971) con- 
cluded that the condition of high adrenocorticotrophic hormone, high glucocorticoid levels associated with avoidance training in intact animals, may retard or enhance the acquisition of these responses, depending on the difficulty of the task or the stage of acquisition. Judging from the frequent reports of training difficulties (e.g., Bolles, 1970; Meyer, Cho, \& Wesemann, 1960) and the problems with competing behaviors (see Hoffman \& Fleshler, 1962), the leverpress is a difficult task for avoidance training. This suggests that high endogenous levels of corticosterone may be antagonistic to leverpress avoidance performance. The 10-sec signal-shock interval used in Experiment 2 may not allow the reported initially high level of corticosterone to adapt out during the course of the avoidance training (Coover et al., 1973; Fjermestad, 1976) and may reinforce its conditioned occurrence as a generalized response to the warning signal and apparatus (Coover et al., 1973), thus inhibiting avoidance responding.

\section{REFERENCE NOTE}

1. Brush, F. R. Personal communication, July 1976.

\section{REFERENCES}

Berger, D. F., \& Brush, F. R. Rapid acquisition of discretetrial lever-press avoidance: Effects of signal-shock interval. Journal of the Experimental Analysis of Behavior, 1975, 24, 227-239.

Bolles, R. C. Species-specific defense reactions and avoidance learning. Psychological Review, 1970, 77, 32-48.

BrUSH, F. R. On the differences between animals that learn and do not learn to avoid electric shock. Psychonomic Science, 1966, 5, 123-124.

Brush, F. R., \& Froelich, J. C. Motivational effect of the pituitary and adrenal hormones. In B. E. Elftheriou \& R. L. Sprott (Eds.), Hormonal correlates of behavior (Vol. 2). New York: Plenum Press, 1975.

Coover, G. D., Ursin, H., \& Levine, S. Plasma corticosterone levels during active-avoidance learning in rats. Journal of Comparative and Physiological Psychology, 1973, 82, 170-174.

Davis, H., Porter, J. W., Livingstone, J., Herrmann, T., MacF AdDen, L. P., \& Levine, S. Pituitary-adrenal activity and leverpress shock escape behavior. Physiological Psychology, 1977, 5, 280-284.

DelfT, A. M. L. VAN. The relationship between pretraining plasma corticosterone levels and the acquisition of an avoidance response in the rat. Progress in Brain Research, 1970, 32, 192-199.
DEVITo, W. Leverpress avoidance and ventral-medial hypothalamic lesions: Relationship to the pituitary-adrenal system in the rat. Unpublished master's thesis, State University of New York College at Cortland, 1979.

Di Giusto, E. L., Cairncross, K., \& King, M. G. Hormonal influences on fear-motivated responses. Psychological Bulletin, $1971,75,432-444$.

Ferguson, E. D. Motivation: An experimental approach. New York: Holt, Rinehart \& Winston, 1976.

Fjermestad, J. L. Corticosterone levels during discriminated leverpress escape versus avoidance learning. Unpublished master's thesis, State University of New York College at Cortland, 1976.

GLick, D., Von Redlich, D., \& Levine, S. Fluorometric determination of corticosterone and cortisol in 0.02-0.05 milliliter of plasma or submilligram samples of adrenal tissue. Endocrinology, 1964, 74, 653-655.

Gray, J. A. The psychology of fear and stress. New York: McGraw-Hill, 1971.

Hoffman, H. S., \& Fleshler, M. The course of emotionality in the development of avoidance. Journal of Experimental Psychology, 1962, 64, 288-294.

Lasser, N. A., Roheim, P. S., Edelstein, D., \& Eder, H. A. Serum lipoproteins of normal and cholesterol-fed rats. Journal of Lipid Research, 1973, 14, 1-8.

LESHNER, A. I. An introduction to behavioral endocrinology. New York: Oxford University Press, 1978.

Levine, S. Stress and behavior. Scientific American, 1971, 224, 26-31.

Meyer, D. R., Cho, C., \& Wesemann, A. F. On problems of conditioned discriminated lever-press avoidance response. Psychological Review, 1960, 67, 224-228.

Natelson, B. H., Krasnegor, N., \& Holaday, J. W. Relations between behavioral arousal and plasma cortisol levels in monkeys performing repeated free-operant avoidance sessions. Journal of Comparative and Physiological Psychology, 1976, 90, 958-969.

Selye, H. The stress of life. New York: McGraw-Hill, 1956.

Solomon, R. L., \& Wynne, L. C. Traumatic avoidance learning: The principles of anxiety conservation and partial irreversibility. Psychological Review, 1954, 61, 353-385.

Weiss, J. M. Somatic effects of predictable and unpredictable shock. Psychosomatic Medicine, 1970, 32, 397-408.

WEISs, J. M. Effects of coping behavior in different warning signal conditions on stress pathology in rats. Journal of Comparative and Physiological Psychology, 1971, 77, 1-13.

Weiss, J. M., McEwen, B. S., Silva, M. T., \& Kalkut, M. F. Pituitary-adrenal influences on fear responding. Science, 1969, 163, 197-199.

Weiss, J. M., McEwen, B. S., Silva, M. T., \& Kalkut, M. Pituitary-adrenal alterations and fear responding. American Journal of Physiology, 1970, 218, 864-868.

Wimersma Greidanus, TJ. B. van. Effect of steroids on extinction of an avoidance response in rats: A structure-activity relationship study. Progress in Brain Research, 1970, 32, 185-191.

(Received for publication September 30, 1980; revision accepted March 12, 1981.) 